\title{
The demonstration that amongs myriads of palliative anticancer plants, there is one that is endowed by a synergical action: The Chenopodium album, that avoids the biotranformation of a benign skin tumor to malignant
}

\section{Lorenzo Martini ${ }^{1}$, Piotr Brzezinski ${ }^{2}$}

${ }^{1}$ University of Siena, Department of Pharmaceutical Biotechnologies, Via A. Moro 2, 53100 Siena, Italy, ${ }^{2}$ Institute of Biology and Environmental Protection, Pomeranian cademy, Slupsk, Poland

Corresponding author: Lorenzo Martini, E-mail: martinil163@libero.it

\begin{abstract}
ABASTRACT
The corresponding Author has experimented upon himself, that is, in corpore vili, the capacity of a common herb (retrievable in all Europe and Americas), the Chenopodium album, the treatment of an abscissed spitz nevus (he had on his shoulder) and has had magnificient results. This herb is endowed by two types of biological principles that can act synergically:the plant is effiecient both for prevention and cure itself of a Spitz nevus and could be proposed for the treatment of other types of skin melanomas in the future.
\end{abstract}

Key words: Spitz nevus; Chenopodium album pulvis; ELM; Venetian talc; Polyphenols

Physiologically, the hormones carry messages to all cells in Man organism, triggering the cells to take action. These messages are carried by blood through the vascular system (arteries, veins and capillaries). The blood carries the other things that cells need to function too. Cells need oxygen and glucose to keep them alive, for example. Blood vessels also carry away waste products and oxygen-poor blood once the cells have used the oxygen in the blood. The lymphatic system helps to clean and drain what we do not need. The lymphatic system is a part of Man's body's defense system, and it drains away bacteria and germs and all cancerous cells (cells where the DNA became foolish and irresponsible) that tend to create growths and then tumors or cancers.

When the lymphatic system is not upset, cellular DNA and all that concerns normal health is regular but cells become abnormal if their DNA - and therefore their "knowledge" - becomes damaged. As long as there are very few abnormal cells and they are kept under control by the immune system, they will not harm man and thus these kinds of "anomalies" may be clinically defined "benign tumors". It is only when these cells start to divide uncontrollably, forming lumps or growths, that it is poissbile to number have one of the more than 200 diseases called cancer. Growths like this are called tumors. The main differences between malignant (cancerous) and benign (noncancerous) tumors are that malignant ones can:

- spread into the surrounding tissue,

- destroy the surrounding tissue, and

- cause other tumors to develop.

Malignant tumors can be life-threatening. But there are also some kinds of cancer that develop so slowly in older people that they do not lead to any problems in their lifetime. 
Benign tumors usually do not cause much damage and are not normally life-threatening. But there is no guarantee: benign growths can become dangerous if they grow a lot, or they might become malignant after a certain amount of time.

If cancer cells start replicating, they do not behave like normal cells. For example, they do not know when to stop replicating and when to die. And they do not always stick together, so they might break away and move through the vascular or lymphatic system and start growing somewhere else in the body.

The Spitz nevus is a rare type of skin mole that usually affects young people and children, but even often elder. Although it can look like a serious form of skin cancer called melanoma, a Spitz nevus lesion isn't considered cancerous, even if in case of immunodeficiency or other pathological conditions, it could grow malignant.

The first step a physician should do is to effectuate the biopsy of the Spitz nevus and control if the nevus is going to become, because of certain causes, malignant.

The biopsy for almost all types of nevi is conducted by the aids of the ELM (Epiluminescence Microscope), ointing the part that is suspected.

If it is completely benign, the second pace is to remove it (abscission).

Afterwards it is important to care that the abscissed nevus (a liminal lesion remains always) can grow malignant anyway.

Apart shamans and sorceresses who may promise the inhibition of the passage from a tumor to become from benign to malignant owing to manifold antiquate and traditional herbs, some of these plants reflects a very important role in this conditions, thanks to the copresence of many biological principles as polyphenols or tannins or glycosides, that can guarantee the impossibility of the tumor to grow malignant, but anyway these constitute a fancy repertoire, that has no scientific approval.

We could observe that the usage of some herbs can be reputed palliative and help to slow the growth of the tumor.

Chenopdium album, amongst many plants containing polyphenols, catechins and tannins, that are reputed antioxidant and sometimes cytotoxic, contains two important principles: an important glycoside:the xyloside and another biological active (see below).

Moreover polyphenols make a major contribution to free radical scavenging capacities. There was a direct relationship between antioxidant activity and total phenolics content in selected herbs, vegetables. Chenopodium album leaves had a relatively high level of total phenolics and extractable condensed tannins which consisted of predominantly procyanidins and prodelphinidins with 2,3-cisstereochemistry. Tannins extracted from leaves, twigs and stem bark all showed very good DPPH radical scavenging activity (IC50 of $56.86,62.31$ and $54.80 \mu \mathrm{g} / \mathrm{ml}$ ) and ferric reducing power (4.28, 3.74 and $4.49 \mathrm{mmol} \mathrm{AAE/g} \mathrm{dried} \mathrm{tannins).}$

The aglycone of this glycoside (xyloside) consists of a hydrophobic compound which aids in carrying the sugar moiety to the Golgi membrane where GAG synthesis takes place, as Proteoglycan (PG) synthesis is initiated by the transfer of D-xylose from UDPxylose to a serine residue in core proteins. This natural primer acts as a template for the assembly of heparin sulfate, heparin, chondroitin sulfate, and dermatan sulfateside chains, depending on the tissue. However, in 1973 it was determined that synthetic B-D-xylosides can prime glycosaminoglycan (GAG) synthesis by substituting for the core xylosylated protein, meanwhile the aglycone moyety is able to stop this process and avoid the growth of dermal cells that could create ex abrupt growth and lumps.

When a malignant tumor is contained within one area and has not spread to the surrounding tissue, the medical term is "carcinoma in situ." If this tumor does not keep growing, that means it is just lying there quietly ("dormant cancer cells"). It is not likely to cause harm unless it starts growing.and eveniences are coexistent.

To keep growing, these tumors start to create their own blood vessels to supply them with the extra oxygen, glucose and hormones they need to survive and keep getting bigger. That process of developing a blood supply system is called angiogenesis (the growth of new blood vessels). Once a tumor does this, it can start to invade the surrounding tissue. This is called an invasive cancer, and it is possible to observe an excrescence, especially in skin carcinomas or nevi.

Moreover manifold lines of evidence suggest that carnosol, contained in the same Chenopodium album, holds the promise of preventing certain types of cancer, 
especially skin melanomas. A remarkable progress has been made in delineating the biochemical mechanisms underlying the chemopreventive effects of carnosol. Results from in vitro cell culture studies as well as animal model experiments have revealed that carnosol inhibits experimentally induced carcinogenesis and exhibits potent anti-oxidative, anti-inflammatory, antiproliferative and apoptosis inducing properties. Moreover, carnosol enhances the sensitivity of chemoresistant cancer cells to chemotherapeutic agents [1].

When a suspect amount of melanocytes or chloasma is detected, the employ of Chenopodium extract could be efficient, but this must be scientifically ascertained in a next future.

It is well known that even in islamic medicine chenopodium was recognized as anticancer remedy [2] .

It must stressed that Cancer is an unbeaten health challenge for the humankind. After striving for decades to find a cancer cure, attention has now been shifted to reduce the morbidity and mortality from cancer by halting the course of tumor development. Numerous bioactive phytochemicals, especially those present in edible and non-edible plant species, have been reported to reduce the risk of many cancers. Multiple lines of evidence suggest that carnosol, a phenolic diterpene contained in Chenopodium album, holds the promise of preventing certain types of cancer. A remarkable progress has been made in delineating the biochemical mechanisms underlying the chemopreventive effects of carnosol. Results from in vitro cell culture studies as well as animal model experiments have revealed that carnosol inhibits experimentally induced carcinogenesis and exhibits potent anti-oxidative, anti-inflammatory, antiproliferative and apoptosis inducing properties. Moreover, carnosol enhances the sensitivity of chemoresistant cancer cells to chemotherapeutic agents.

Epidemiological, clinical and laboratory studies have implicated solar ultraviolet (UV) radiation in various skin diseases including premature aging of the skin and melanoma and nonmelanoma skin cancers. Chronic UV radiation exposure-induced skin diseases or skin disorders are caused by the excessive induction of inflammation, oxidative stress and DNA damage, etc.. The use of chemopreventive agents, such as plant polyphenols, to inhibit these events in UV-exposed skin is gaining attention. Chemoprevention refers to the use of agents that can inhibit, reverse, or retard the process of these harmful events in the UV-exposed skin. A wide variety of polyphenols or phytochemicals, most of which are dietary supplements, have been reported to possess substantial skin photoprotective effects. Presently the photoprotective agents from some selected polyphenols, are considered as valuable, like green tea polyphenols, grape seed proanthocyanidins, resveratrol, silymarin and genistein, on UV-induced skin inflammation, oxidative stress, and DNA damage, etc., with a focus on mechanisms underlying the photoprotective effects of these polyphenols. The laboratory studies conducted in animal models, suggest that these polyphenols have the ability to protect the skin from the adverse effects of UV radiation, including the risk of skin cancers. It is suggested that polyphenols may favorably supplement sunscreens protection, and may be useful for skin diseases associated with solar UV radiation-induced inflammation, oxidative stress and DNA damage.

Cancer of the skin is characterized by an imbalance towards too little apoptosis, or too much cell proliferation and survival in the epidermis $[3,4]$. Although UV radiation is the leading cause of skin cancer, other causative agents include viruses, mutagens in food, mutagens in chemicals and genetic susceptibility $[5,6]$. Skin cancer can be prevented by controlling, or eliminating these causative agents. Skin cancer can be effectively removed by hindering blood supply to the tumor (anti-angiogenesis), which curbs tumor growth and enhances patient survival. Most cancer cells develop ways to evade apoptosis, or exhibit defective apoptosis mechanisms, thus allowing uncontrollable cell development [2]. The apoptosis process is therefore the major target of anti-cancer chemotherapeutics or natural drugs (Box 1).

Finally laboratory studies conducted in animal models, suggest that these polyphenols have the ability to protect the skin from the adverse effects of UV radiation, including the risk of skin cancers. It is suggested that polyphenols may favorably supplement sunscreens protection, and may be useful for skin diseases associated with solar UV radiation-induced inflammation, oxidative stress and DNA damage.

The Spitz nevus was discovered on right shoulder by in Martini and since We are doctor and can follow the Sir Percival ethical rights (that permits the experimentations on oneself with no consense of whichever medical ethical committee) We underwent to a ELP biopsy and have the melanoma abscissed.

After two days We began the following treatment: 
Quercetin, Kaempferol, EGCG, Apigenin, $\beta$-carotene, Fucoxanthin, Vitamin C, Ganoderma lucidum extract, Coriolus versicolor extract, Resveratrol, Curcumin, Sulforaphane, Melaleuca alternifolia extract, Zingiber officinale extract, Withaferin A from Withania somnifera, Eupatilin from Artemisia, Galangin from Alpinia officinarum, Kaempferol, Apigenin, Vitamin A, Vitamin C, Vitamin D, Vitamin E, Ganoderma lucidum extract, Coriolus versicolor extract, Hypericum perforatum extract, Melaleuca alternifolia extract, Calendula officinalis extract, Emodin from Aloe, Eupatilin from Artemisia, Alpinia oxyphylla extract, Alpinia galangal extract, Amentoflavone, Hinokiflavone, $\beta$-carotene, Fucoxanthin, Resveratrol, Sulforaphane, Withania somnifera extract, Viscum album extract, Calendula officinalis extract, Carnosol from Chenopodium album, Ursolic acid from Rosmarinus officinalis.

Box 1: List of drugs.

a powder made of $50 \%$ chenopodium album pulvis and $50 \%$ venetian talc.

The treatment has lasted 14 days.

After this period We have prayed a friend of mine (dermatologist) to observe by a dermascope (DinoLite) to state the condition of my skin.

No lesion or shadow of past scare was visible at all.

No itching, no redness and no skinmanifestation was detectable.

Notwithstanding the avalanche of herbs that promise the prevention and the complete cure of a skin melanoma, I propose Chenopodium album pulvis to avoid that a tumor (considered after a biopsy by ELP benign) becomes malignant.

Even rosemary (Rosmarinus off. contains great percentages of carnosol, but does not contain absolutely the xyloside.

One is excellent to prevent and the other is exceptional to treat eventual transformation of a benign skin cancer to malignant.

\section{REFERENCES}

1. Conroy ML, Davis KR, Embree JL, Madara B, Magaletto P, Roach RR. Sauls: Carnosol: A promising anti-cancer and antiinflammatory agent. Cancer Lett. 2012;305:1.

2. Emami SA, Sahebkar A, Tayarani-Najaran N, Tayarani-Najaran Z. Cancer and its Treatment in Main Ancient Books of Islamic Iranian Traditional Medicine (7th to 14th Century AD). Iran Red Crescent Med J. 2012;14:747-57.

3. Laniauskaite I, Ožalinskaite A, Strupaite R, Bylaite M. Skin cancer knowledge, attitude and behavior towards sun exposure among young adults in Lithuania. Our Dermatol Online. 2011;2:189-95.

4. Cengiz FP, Sallahoglu K, Emiroglu N, Onsun N. The number of dysplastic and common nevi in patients with malignant melanoma. Our Dermatol Online. 2018;9:128-30.

5. Chinembiri TN, du Plessis LH, Gerber M, Hamman JH, du Plessis J. Review of natural compounds for potential skin cancer treatment. Molecules. 2014;19:11679-721.

6. Marks VJ, Hanson NW. Non-melanoma skin cancer. In Sauer's Manual of Skin Diseases; Hall, B.J., Hall, J.C., Eds.; Wolters Kluwer Health: Philadelphia, PA, USA, 2010; Volume 10, pp. 305-312.

Copyright by Lorenzo Martini, et al. This is an open access article distributed under the terms of the Creative Commons Attribution License, which permits unrestricted use, distribution, and reproduction in any medium, provided the original author and source are credited.

Source of Support: Nil, Conflict of Interest: None declared. 